\title{
A Cooperative Monitoring Approach of Hydro Turbine Generator Sets based on Multi-agent Technology
}

\author{
Fanwu Chu ${ }^{1, a^{*}}$, Zhaohui $\mathrm{Li}^{1, \mathrm{~b}}$ \\ ${ }^{1}$ Huazhong University of Science and Technology, Wuhan, 430074, China \\ aemail: chufw@qq.com, bemail:proflzhh@126.com
}

Keywords: Hydro Turbine Generator Set; Condition monitoring; Multi-agent Technology; Cooperative Monitoring; health condition assessment

\begin{abstract}
Hydro Turbine Generator Set (HTGS) is an organic entity though it is composed by numerous equipments. However, almost all of those monitors applied in plant operate independently. Data and information from those independent systems lack correlation. This situation makes it difficult to determine whether or not there are potential faults. This paper proposes a cooperative monitoring approach based on multi-agent technology. A modified agent reference model is listed and the framework of the whole monitoring system is introduced. Then cooperative work process based on blackboard model and the cooperation mechanism based on operation condition synchronization (OCS) and event synchronization (ES) technology employed in this approach are also introduced. Application shows that this new approach is able to overcome the deficiency of single independent monitoring system for the interpretation of detected fault and health condition assessment.
\end{abstract}

\section{Introduction}

With the increase of the capacity of hydro turbine generator sets (HTGSs) and fierce market competition, great demands on the condition monitoring technology of hydro turbine generator sets (HTGSs) are put forward. There have been many specialized monitoring and diagnosis systems [1-4] operating in isolation applied in power plant around the world.

HTGS is an organic entity though it is composed by numerous equipments [5]. Operation of single equipment is closely related with other equipments. Data and information from those independent monitoring systems lack correlation. Generally, the combined effect created by the corporation of two or more persons is greater than the sum of their individual effects. So the idea of cooperation has been paid more and more attention. Currently, multi-agent technology [6] is growing rapidly which is becoming an important development direction of artificial intelligence. Now it has been widely applied in the field of maintenance decision support [7], condition monitoring [8] [9] and charging control strategy of electric vehicle [10]. Application of multi agent technology to HTGS condition monitoring will have important significance.

Therefore, this paper regards those independent systems as members and gives the reference model of a modified agent. Then the framework of a coordinate monitoring system of HTGS, which takes advantage of agents as team members, is also designed and the division of labor and responsibilities of each agent in this monitoring team is detailed. In addition, this paper gives a cooperative working mode based on blackboard model and describes the cooperation trigger mechanism based on operation condition synchronization (OCS) and event synchronization (ES) technology. This approach strength the information sharing and data correlation among agents and let them cooperate closely with each other and work as an efficient monitoring team. 


\section{Reference Model of Modified Agent}

The reference model of modified agent is established based on the understanding of basic characteristics and structure of agent, and functional requirements of cooperative monitoring system. Modified agent includes four modules, namely the target analysis module (TAM), the condition analysis module (CAM), the state and fault analysis module (S\&FAM), and the decision analysis module (DAM), as shown in Fig. 1.

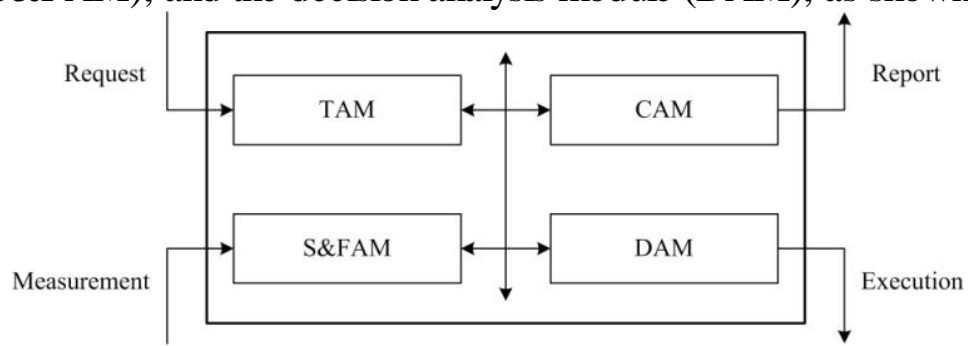

Fig. 1 Reference model of modified agent

TAM accepts the request information from other agent, combines with its own and other modules operating condition, and analyzes the rationality of the request and whether the device is able to achieve the desired target.

CAM produces a comprehensive report on the device state or system characteristics based on the analysis results obtained from TAM, and then sends the report to the request agent.

S\&FAM checks the validity of measurement results froma technical point, operational point and functional point in order to determines whether the observation is valid or not. It also diagnoses and analyzes the faulttype and fault location.

DAM supports to select control strategy to achieve effective goals based on the analysis results from S\&FAM.

\section{The Framework of System}

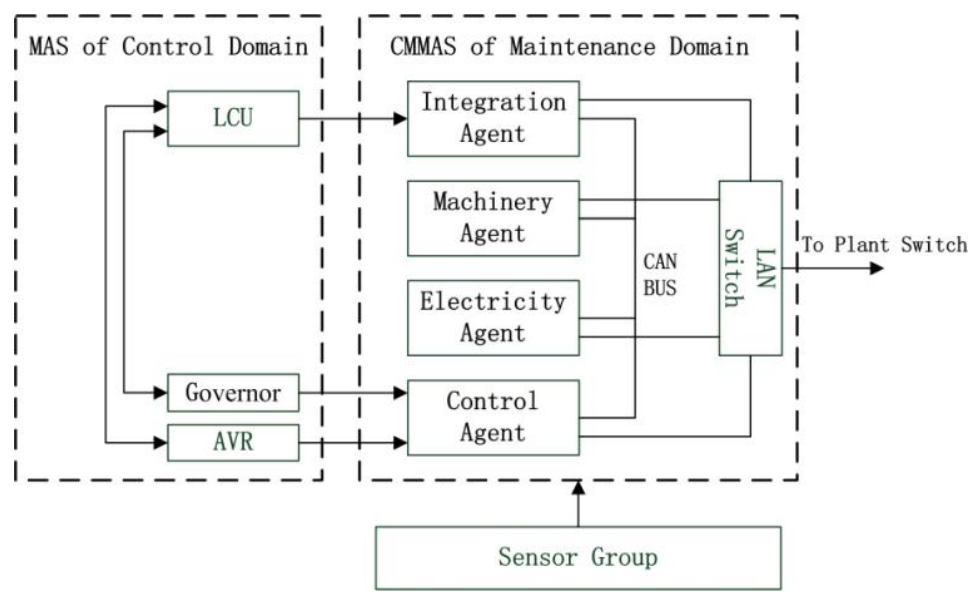

Fig. 2 The framework of cooperative monitoring system

Framework of this system for HTGS is shown in Fig. 2. There are two MAS under this framework: cooperative monitoring multi agent system (CMMAS) of maintenance domain and MAS of control domain. CMMAS contains control agent, electricity agent, machinery agent, and integration agent. Each agent is joined into a real-time CAN bus and industrial Ethernet, that make it easy to share information, transfer data, and realize the interaction, collaboration, and cooperation among team members. MAS of control domain contains local control unit (LCU), automatic voltage regulator (AVR) and Governor. With the completion of their own control tasks, these agents transfer monitoring data of themselves from control domain to maintenance domain unidirectional through secure technology of communication widely used in plant, which makes it possible to share data safely between agents of different domains. 


\section{Agent Work Division}

Each agent mentioned above is a member of the HTGS's monitoring team and the work divisions of agent in this monitoring team are as follows:

1) Control agent. It is responsible for obtaining operating states data from GOV and AVR in MAS of controldomain, acquiring data from sensor group with high precision to meet the requirements of analysis and diagnosis and identifies HTGS operating conditions.

2) Electricity agent. It mainly acquires states data of generator and transformer, such as generator partial discharge amplitude, air gap between stators and rotors, magnetic field intensity, transformer core grounding current, etc. On the basis of monitoring those states, it is responsible for detecting faults and analyzing insulation conditions of generator and transformer.

3) Machinery agent. It is responsible for acquiring data of mechanical system operating states including water pressure, vibration, swing, water pressure pulsation, turbine cavitations, etc. Based on those motion states, it is able to analyze stability health condition of HTGS.

4) Integration agent. It works as the data center of this monitoring system, which is responsible for collecting data from control domain and maintenance domain and storing them for further analysis.

\section{Cooperative Work Process based on Blackboard Model}

Blackboard model [11] consists of knowledge sources (KSs), blackboard, and controller (shown in Fig. 3). Independent experts, which have different specialized knowledge to solve different domain problem, are called $\mathrm{KSs}$, namely agents mentioned above. The blackboard is a problem solving shared workplace which is responsible for storing data and information needed by KSs. Controller is able to resolve conflicts between various KSs. According to some cooperation mechanisms, controller selects and activates appropriate agents to make those agents react to the change of blackboard.

In blackboard model, each agent can get information from blackboard when they work together to solve problem collaboratively. When problems and initial data are displayed on the blackboard, problem solving begins. All agents look at blackboard and look for opportunities to solve problems. If an agent gets enough information and data from blackboard to support its further problem solving, it will post the results on blackboard. This additional information may contribute to make other agents continue solving problem. This process will be repeated until the problem is completely solved.

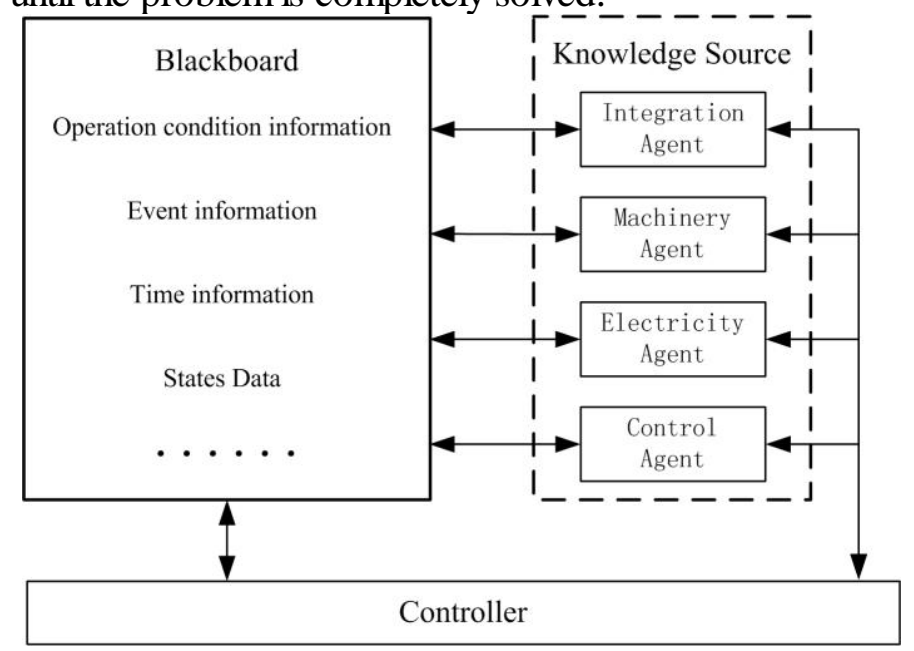

Fig. 3 Cooperate work process based on Blackboard model 


\section{Cooperation Mechanism based on Operation Condition Synchronization}

Operating states and health condition of HTGS are closely associated with the set operation condition. For example, the peak values of swing and vibration of HTGS under load rejection condition are much larger than load stable condition. Therefore, synchronously acquiring and analyzing data among different agents is very important to fault diagnosis. The OCS process of CMMAS is as follows:

1) Control agent obtains data of operation condition states (including water head, active power, reactive power, frequency, stator voltage, stator current, guide vane opening, wheel opening etc.) by the way of acquisition and communication.

2) Then Control agent uses Petri Net model and states data to distinguish operation conditions of HTGS in real time and then post the Operation Condition Information (OCI, including code, name, start time and end time of the current operation condition) on blackboard through CAN bus every 50 milliseconds.

3) Other agents get OCI fromblackboard through CAN bus. Integration agent stores the OCI as a record in database and other agents upload operating states data to Integration agent of this condition process based on the specific operation condition which are stored in Integration agent.

Through the above steps, each agent in CMMAS can know the current operation condition in real time so that they can monitor operating state, store states data and analyze device health condition synchronously.

\section{Cooperation Mechanism based on Event Synchronization}

Various types of operations and abnormalalarms are collectively referred as event in CMMAS. During the event process, operating states data is important to fault diagnosis. The ES process of CMMAS is as follows:

1) Each agent distinguishes the state of switch quantities and judges whether the operation events and alarm events happened or not in real time. At the same time, each agent detects whether there is an abnormality in monitoring objects using threshold detection method based on the state data and performance data. They generate an alarm event when data is out of threshold.

2) Once event is detected, each agent posts the event information (including code, name, start time and end time of current event) on blackboard through CAN bus.

3) Similar with the OCS, other agents get event information from blackboard. Integration agent stores event information as a record in database and other agents upload operating states data to Integration agent of this process based on the specific event which are stored in Integration agent.

Through the above steps, each agent in CMMAS can know current event in real time so that they can monitor operating state, store states data and analyze device health condition synchronously.

\section{Application Notes}

Currently this approach is applied in Hydropower plant Optimal Maintenance Information System (HOMIS) [12], which has been employed by Gezhouba Power Plant to evaluate the health condition of all 22 HTGSs, and achieved good effects.

Fig. 4 shows the trend curve of upper guide swing of HTGS when it changes its active power output. Curves in Fig. 4 are: (a) frequency (Hz), (b) active power (MW), (c) guide vane opening (\%), (d) X upper guide swing $(\mu \mathrm{m})$, (e) Y upper guide swing $(\mu \mathrm{m})$, and $\mathrm{x}$-axis is time(M/D/Y H:M:S). The machine was a $13.8 \mathrm{kV}, 146 \mathrm{MVA}$ HTGS. Those data are obtained by the proposed approach. 


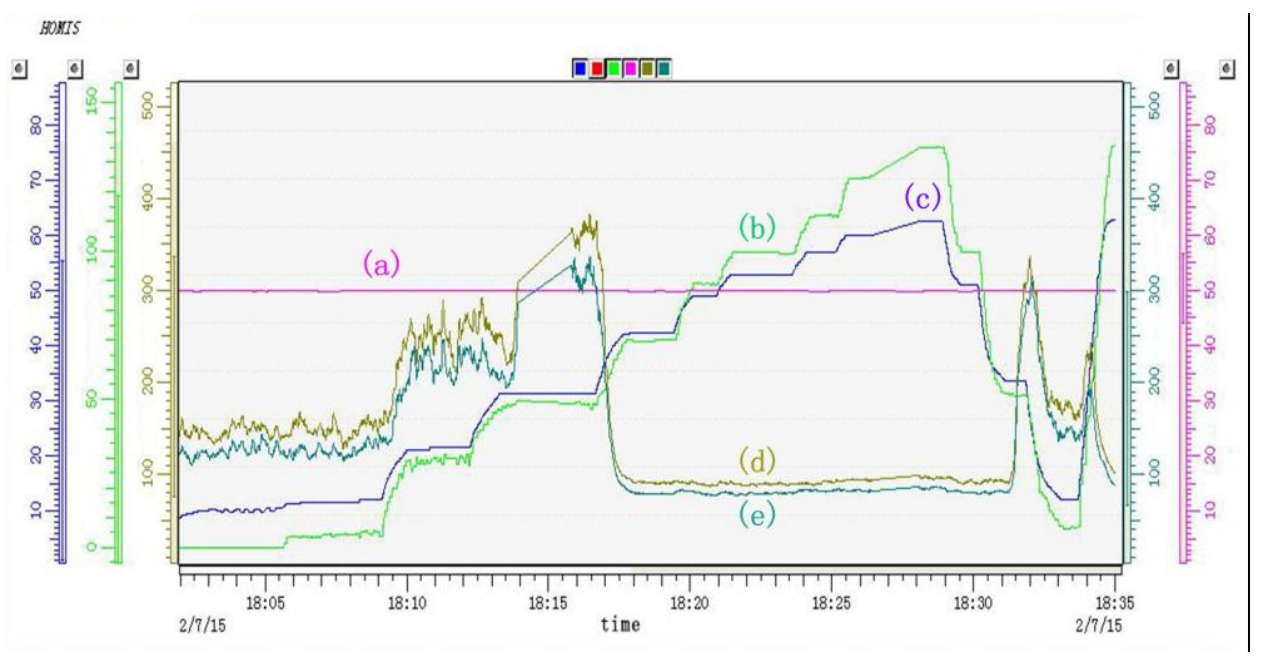

Fig. 4 Curve of upper guide swing when HTGS changes its active power output

The amplitude of upper guide swings becomes large when active power changes from 0 to $50 \mathrm{MW}$ and achieves the peak value when active power is 50MW. Then the amplitude of upper guide swings becomes small with the increase of active power and keeps in a small stable value. From Fig. 4 we know that the stability of this set goes bad when its active power changes between 30 and $60 \mathrm{MW}$ and this range is the so called unstable operation region which is usually advised to forbidden operating because the set will be damaged seriously under this condition.

\section{Conclusions}

This paper has proposed a cooperative monitoring approach based on multi-agent technology. It regards different monitoring systems operating in isolation as members and combines them into a monitoring team. With cooperation mechanisms based on operation condition synchronization and event synchronization, this approach achieves a comprehensive monitoring of HTGS which assists staffs make an accurate interpretation of detected faults and assess health condition of HTGS. Application shows that Gezhouba Power Plant benefits from this cooperative monitoring approach to HTGS monitoring and health condition assessment.

\section{References}

[1] Stone G C. Advancements during the past quarter century in on-line monitoring of motor and generator winding insulation[J]. IEEE Transactions on Dielectrics \& Electrical Insulation, 2002, 9(5):746 - 751.

[2] Liu Ming-Jun, Han Di, and Li Zhao-Hui. Online UHF PD Monitoring for Transformers Under the Integrated Framework of HOMS. High Voltage Engineering, 2010, 36 (8):1975-1980.

[3] Huixuan Shi, Zhaohui Li, Yaxiong Bi, An On-line Cavitation Monitoring System for Large Kaplan Turbines [C]. IEEE PES General Meeting, 2007: 1-6.

[4] Y.Han, Y.H.Song. Condition Monitoring Techniques for Electrical Equipment-A Literature Survey. IEEE Transactions on Power Delivery, 2003, 18(1): 4-13.

[5] XIE Guocai, LI Zhaohui. Integrated monitoring of hydroelectric generators based on Community Intelligence [J]. Electric Power Automation Equipment, 2013, 33(1): 153-159

[6] Wooldridge M. Intelligent agents: theory and practice[J]. Knowledge Engineering Review, 1995, 10(2):115--152. 
[7] Liu Zhiyong, Jia Xisheng, Rong Liqiang. Study on Maintenance Decision-making Technology Based on Multi-Agent in PHM [C]. Third International Conference on Measuring Technology and Mechatronics Automation, 2011:495-498.

[8] McArthur S D J, Strachan S M, Jahn G. The design of a multi-agent transformer condition monitoring system[J]. IEEE Transactions on Power Systems, 2004, 19(4):1845 - 1852.

[9] McArthur S D J, Strachan S M, Jahn G. The design of a multi-agent transformer condition monitoring system[J]. IEEE Transactions on Power Systems, 2004, 19(4):1845 - 1852.

[10] XU Shaolun, YAN Zheng, FENG Donghan, Cooperative charging control strategy of electric vehicle based on multi-agent[J]. Electric Power Automation Equipment, 2014, 34(11): 7-13

[11] Mcmanus J W, Bynum W L. Design and analysis techniques for concurrent blackboard systems[J]. IEEE Transactions on Systems Man \& Cybernetics Part A Systems \& Humans, 1996, 26(6):669 - 680.

[12] Zhaohui LI, Youzhong AI, Huixuan SHI. Optimal Maintenance Information System of Gezhouba Hydro Power Plant. In Proceedings of 2007 IEEE PES General Meeting, 2007: 1-5. 